\title{
Change in incidence of clinic visits for all-cause and rotavirus gastroenteritis in young children following the introduction of universal rotavirus vaccination in Israel
}

\author{
K Muhsen ${ }^{12}$, G Chodick ${ }^{12} 3$, S Goren ${ }^{1}$, E Anis ${ }^{4}$, T Ziv-Baran ${ }^{1}$, V Shalev ${ }^{13}$, D Cohen ${ }^{1}$ \\ 1. Department of Epidemiology and Preventive Medicine, School of Public Health, Sackler Faculty of Medicine, Tel Aviv \\ University, Tel Aviv, Israel \\ 2. These authors contributed equally to the article \\ 3. Medical Division, Maccabi Health Services, Tel Aviv, Israel \\ 4. Division of Epidemiology, Ministry of Health, Jerusalem, Israel
}

Correspondence: Khitam Muhsen (kmuhsen@post.tau.ac.il)

Citation style for this article:

Muhsen K, Chodick G, Goren S, Anis E, Ziv-Baran T, Shalev V, Cohen D. Change in incidence of clinic visits for all-cause and rotavirus gastroenteritis in young children following the introduction of universal rotavirus vaccination in Israel. Euro Surveill. 2015;20(42):pii=30045. DOI: http://dx.doi.org/10.2807/1560-7917. ES.2015.20.42.30045

Article submitted on 19 December 2014 / accepted on 17 March 2015 / published on 22 Ocotber 2015

Both rotavirus vaccines RotaTeq and Rotarix were efficacious against severe rotavirus gastroenteritis in clinical trials; yet real-world data on the effect of rotavirus vaccines on mild to moderate disease are limited. We used a large computerised database of Maccabi Health Services Health Maintenance Organisation (HMO), the second largest HMO in Israel covering $\mathbf{2 5} \%$ of the Israeli population, to compare the incidence of acute gastroenteritis (AGE) clinic visits in community settings $(n=302,445)$ before $\left(2005^{-10}\right)$ and after (2011-13) the introduction of universal rotavirus immunisation in Israel. We retrieved laboratory results of rotavirus antigen tests $(n=18,133)$ and using a weighted analysis, we estimated the impact of rotavirus immunisation on the disease burden of rotavirus $A G E$ clinic visits. Following the introduction of universal rotavirus immunisation, the typical winter peaks of rotavirus AGE were substantially lower and significant reductions of $14.8 \%$ (95\% confidence interval (CI): 13.5-16.1) in all-cause AGE clinic visits and of $59.7 \%$ (95\% Cl: 59.8-62.6) in rotavirus AGE clinic visits were observed. The decrease was observed in all age groups, but it was greater in children aged o to 23 months than those aged 24 to 59 months. Continued rotavirus laboratory surveillance is warranted to monitor the sustainability of these changes.

\section{Introduction}

Rotavirus is the leading cause of severe acute gastroenteritis (AGE) in young children worldwide [1-3], accounting for 15 to $35 \%$ of AGE outpatient visits, 25 to $55 \%$ of severe $\mathrm{AGE}$ cases requiring hospitalisation, and $5 \%$ of the global mortality in children under five years $[3,4]$. The majority of deaths caused by rotavirus occur in developing countries $[1,3,4]$, while in industrialised countries, rotavirus gastroenteritis is associated with high healthcare costs $[5,6]$ as well as non-medical costs [7].

The new rotavirus vaccines RotaTeq (Merck) and Rotarix (GlaxoSmithKline) have shown an efficacy of 85 to $98 \%$ against severe rotavirus gastroenteritis in trials conducted in the Americas and Europe $[8,9]$, and were introduced into routine vaccination programmes in the United States, Latin American countries, Australia [1012] and few European countries [13,14]. Post-marketing studies have shown that rotavirus vaccines are highly effective (>80\%) in preventing hospitalisations for rotavirus gastroenteritis $[12,15-18]$ in high and middle income countries, although a lower effectiveness was reported in some settings [12]. In these countries, significant reductions $(49-89 \%)$ in rotavirus gastroenteritis hospitalisations of children younger than five years were documented in the period after introduction of rotavirus vaccination compared with the pre-vaccination era [12]. Furthermore, fatality from diarrhoeal diseases in young children declined substantially in Mexico [19] and Brazil [20] following the introduction of rotavirus vaccination.

Severe rotavirus disease resulting in hospitalisations and deaths comprises only the tip of the iceberg of the rotavirus disease burden [3]. Only limited evidence, mainly from the United States [21,22], exists on the impact of rotavirus vaccination on mild to moderate gastroenteritis in community and outpatients settings.

In a previous study, we found that rotavirus was the leading cause of AGE hospitalisations in the pre-vaccination period (2007-08), accounting for $39 \%$ of all gastroenteritis hospitalisations among children under the age of five years in northern Israel, with typical winter 
Incidence of clinic visits due to acute gastroenteritis in children aged 0-59 months, Maccabi Health Services, Israel, 2005-14 $(\mathrm{n}=302,445)$

\begin{tabular}{|c|c|c|c|c|c|c|c|c|c|c|}
\hline \multirow[b]{2}{*}{ Year } & \multirow[b]{2}{*}{$\begin{array}{c}\text { Age } \\
\text { (months) }\end{array}$} & \multicolumn{3}{|c|}{ All } & \multicolumn{3}{|c|}{ Male } & \multicolumn{3}{|c|}{ Female } \\
\hline & & Visits & opulation & $\mathrm{IR} / 100(95 \% \mathrm{Cl})$ & Visits & Population & $\mathrm{IR} / 100(95 \% \mathrm{Cl})$ & Visits & Population & IR/100 (95\% Cl) \\
\hline \multirow{3}{*}{2005} & $0-59$ & 27,728 & 182,647 & $15.2(15.0-15.4)$ & 15,455 & 94,659 & $16.3(16.1-16.6)$ & 12,273 & 87,988 & $14.0(13.7-14.2)$ \\
\hline & $0-23$ & 18,313 & 72,119 & $25.4(25.1-25.7)$ & 10,121 & 37,308 & $27.1(26.7-27.6)$ & 8,192 & 34,811 & $23.5(23.1-24.0)$ \\
\hline & $24-59$ & 9,415 & 110,528 & $8.5(8.4-8.7)$ & 5,334 & 57,351 & $9.3(9.1-9.5)$ & 4,081 & 53,177 & $7.7(7.5-7.9)$ \\
\hline \multirow{3}{*}{2006} & $0-59$ & 37,664 & 182,473 & $20.6(20.5-20.8)$ & 20,822 & 94,484 & $22.0(21.7-22.3)$ & 16,842 & 87,989 & $19.1(18.9-19.4)$ \\
\hline & $0-23$ & 24,503 & 70,778 & $34.6(34.3-35.0)$ & 13,518 & 36,640 & $\begin{array}{c}36.9 \\
(36.4-37.4) \\
\end{array}$ & 10,985 & 34,138 & $32.2(31.7-32.7)$ \\
\hline & $24-59$ & 13,161 & 111,695 & $11.8(11.6-12.0)$ & 7,304 & 57,844 & $12.6(12.4-12.9)$ & 5,857 & 53,851 & $10.9(10.6-11.1)$ \\
\hline \multirow{3}{*}{2007} & $0-59$ & 32,271 & 182,732 & $17.7(17.5-17.8)$ & 17,787 & 94,444 & $18.8(18.6-19.1)$ & 14,484 & 88,288 & $16.4(16.2-16.7)$ \\
\hline & $0-23$ & 21,213 & 70,419 & $30.1(29.8-30.5)$ & 11,708 & 36,394 & $32.2(31.7-32.7)$ & 9,505 & 34,025 & $27.9(27.5-28.4)$ \\
\hline & $24-59$ & 11,058 & 112,313 & $9.9(9.7-10.0)$ & 6,079 & 58,050 & $10.5(10.2-10.7)$ & 4,979 & 54,263 & $9.2(8.9-9.4)$ \\
\hline \multirow{3}{*}{2008} & $0-59$ & 36,634 & 184,461 & $19.9(19.7-20.0)$ & 20,025 & 95,273 & $21.0(20.8-21.3)$ & 16,609 & 89,188 & $18.6(18.4-18.9)$ \\
\hline & $0-23$ & 24,223 & 72,452 & $33.4(33.1-33.8)$ & 13,165 & 37,324 & $35.3(34.8-35.8)$ & 11,058 & 35,128 & $31.5(31.0-32.0)$ \\
\hline & $24-59$ & 12,411 & 112,009 & $11.1(10.9-11.3)$ & 6,860 & 57,949 & $11.8(11.6-12.1)$ & 5,551 & 54,060 & $10.3(10.0-10.5)$ \\
\hline \multirow{3}{*}{2009} & $0-59$ & 27,581 & 187,141 & $14.7(14.6-14.9)$ & 15,104 & 96,250 & $15.7(15.5-15.9)$ & 12,477 & 90,891 & $13.7(13.5-14.0)$ \\
\hline & $0-23$ & 18,992 & 74,487 & $25.5(25.2-25.8)$ & 10,385 & 38,050 & $27.3(26.9-27.7)$ & 8,607 & 36,437 & $\begin{array}{c}23.6 \\
(23.2-24.0)\end{array}$ \\
\hline & $24-59$ & 8,589 & 112,654 & $7.6(7.5-1.8)$ & 4,719 & 58,200 & $8.1(7.9-8.3)$ & 3,870 & 54,454 & $7.1(6.9-7.3)$ \\
\hline \multirow{3}{*}{2010} & $0-59$ & 40,964 & 192,762 & $21.3(21.1-21.4)$ & 22,363 & 99,154 & $22.6(22.3-22.8)$ & 18,601 & 93,608 & $19.9(19.6-20.1)$ \\
\hline & $0-23$ & 25,980 & 78,021 & $33.3(33.0-33.6)$ & 14,187 & 40,078 & $\begin{array}{c}35.4 \\
(34.9-35.9)\end{array}$ & 11,793 & 37,943 & $31.1(30.6-31.6)$ \\
\hline & $24-59$ & 14,984 & 114,741 & $13.1(12.9-13.3)$ & 8,176 & 59,076 & $13.8(13.6-14.1)$ & 6,808 & 55,665 & $12.2(12.0-12.5)$ \\
\hline \multirow{3}{*}{2011} & $0-59$ & 27,922 & 195,670 & $14.3(14.1-14.4)$ & 15,527 & 100,409 & $15.2(15.0-15.4)$ & 12,647 & 95,261 & $13.3(13.1-13.5)$ \\
\hline & $0-23$ & 17,886 & 78,886 & $22.7(22.4-23.0)$ & 9,644 & 40,591 & $\begin{array}{c}23.8 \\
(23.4-24.2) \\
\end{array}$ & 8,242 & 38,295 & $21.5(21.1-21.9)$ \\
\hline & $24-59$ & 10,036 & 116,784 & $8.6(8.4-8.8)$ & 5,631 & 59,818 & $9.4(9.2-9.7)$ & 4,405 & 56,966 & $7.7(7.5-8.0)$ \\
\hline \multirow{3}{*}{2012} & $0-59$ & 30,753 & 195,012 & $15.8(15.6-15.9)$ & 16,709 & 99,961 & $16.7(16.5-17.0)$ & 14,044 & 95,051 & $14.8(14.6-15.0)$ \\
\hline & $0-23$ & 19,215 & 76,127 & $25.2(24.9-25.6)$ & 10,440 & 39,023 & $26.8(26.3-27.2)$ & 8,775 & 37,104 & $23.7(23.2-24.1)$ \\
\hline & $24-59$ & 11,538 & 118,885 & $9.7(9.5-9.9)$ & 6,269 & 60,938 & $10.3(10.1-10.5)$ & 5,269 & 57,947 & $9.1(8.9-9.3)$ \\
\hline \multirow{3}{*}{2013} & $0-59$ & 32,266 & 194,608 & $16.6(16.4-16.8)$ & 17,729 & 99,951 & $17.7(17.5-18.0)$ & 14,537 & 94,657 & $15.4(15.1-15.6)$ \\
\hline & $0-23$ & 20,764 & 75,713 & $27.4(27.1-27.7)$ & 11,522 & 38,997 & $29.6(29.1-30.0)$ & 9,242 & 36,716 & $25.2(24.7-25.6)$ \\
\hline & $24-59$ & 11,502 & 118,895 & $9.7(9.5-9.8)$ & 6,207 & 60,954 & $10.2(10.0-10.4)$ & 5,295 & 57,941 & $9.1(8.9-9.4)$ \\
\hline $2014^{\mathrm{a}}$ & $0-59$ & 8,662 & 194,281 & a & 4,570 & 99,916 & $\mathrm{a}$ & 4,092 & 94,365 & a \\
\hline
\end{tabular}

$\mathrm{Cl}$ : confidence intervals; IR: incidence rate.

a Data up to 16 May 2014, therefore the incidence rate was not calculated for 2014.

seasonality and peak incidence around December [23]. In mid-2007, both RotaTeq and Rotarix were licensed in Israel and parents could purchase the vaccine through the Health Maintenance Organisation (HMO) with partial reimbursement [24]. In December 2010, the Ministry of Health included three doses of RotaTeq in the childhood national immunisation programme (NIP) at ages 2, 4, and 6 months.

We aimed to estimate the impact of the universal rotavirus immunisation programme on the burden of mild to moderate AGE in children younger than five years by comparing the incidence and seasonality of clinic visits for all-cause AGE and for rotavirus AGE in community settings before (2005-10) and after (2011-13) the inclusion of universal rotavirus vaccination.

\section{Methods}

Study population and design

A retrospective study was conducted using the computerised database of Maccabi Health Services (MHS), a health maintenance organisation (HMO) with 2 million members in Israel, covering one quarter of the Israeli population. All Israeli citizens have health insurance according to the National Health Insurance Law introduced in 1995, consequently all citizens have access to healthcare. Included in the study population were all children younger than five years and insured in the MHS HMO during the study period from 1 January 2005 through 16 May 2014. The total number of children ranged from 182,473 to 195,670 in those years. Access 
TABLE 2

Incidence of clinic visits due to all-cause acute gastroenteritis before (2005-10) and after (2011-13) introduction of universal rotavirus vaccination, by age group, Maccabi Health Services, Israel $(n=64,121)$

\begin{tabular}{|c|c|c|c|c|c|c|c|c|}
\hline \multirow[b]{2}{*}{$\begin{array}{l}\text { Age } \\
\text { (months) }\end{array}$} & \multicolumn{3}{|c|}{$\begin{array}{l}\text { Before universal vaccination } \\
\qquad(2005-10)\end{array}$} & \multicolumn{3}{|c|}{$\begin{array}{l}\text { Universal vaccination era } \\
\qquad(2011-13)\end{array}$} & \multicolumn{2}{|c|}{ Reduction } \\
\hline & Visits/year & Population & $\mathrm{IR} / 100(95 \% \mathrm{Cl})$ & $\begin{array}{l}\text { Visits/ } \\
\text { year }\end{array}$ & Population & $\mathrm{IR} / 100(95 \% \mathrm{Cl})$ & $\begin{array}{c}\text { Risk difference } \\
\text { per } 100 \\
(95 \% \mathrm{Cl})\end{array}$ & $\begin{array}{l}\text { RR reduction } \\
\quad(95 \% \mathrm{Cl})\end{array}$ \\
\hline $0-11$ & 8,967 & 35,495 & $\begin{array}{c}25.3 \\
(24.8-25.7) \\
\end{array}$ & 7,639 & 37,572 & $\begin{array}{c}20.3 \\
(19.9-20.7) \\
\end{array}$ & $\begin{array}{c}4.9 \\
(4 \cdot 3-5 \cdot 5) \\
\end{array}$ & $\begin{array}{c}19.5 \\
(17.1-21.9) \\
\end{array}$ \\
\hline $12-23$ & 13,237 & 37,551 & $\begin{array}{c}35.3 \\
(34.8-35.7) \\
\end{array}$ & 11,649 & 39,337 & $\begin{array}{c}29.6 \\
(29.2-30.1) \\
\end{array}$ & $\begin{array}{c}5.6 \\
(5.0-6.3) \\
\end{array}$ & $\begin{array}{c}16.0 \\
(14.1-17.9) \\
\end{array}$ \\
\hline $24-35$ & 6,511 & 37,669 & $\begin{array}{c}17.3 \\
(16.9-17.7) \\
\end{array}$ & 5,989 & 39,611 & $\begin{array}{c}15.1 \\
(14.8-15.5) \\
\end{array}$ & $\begin{array}{c}2.2 \\
(1.6-2.7) \\
\end{array}$ & $\begin{array}{c}12.5 \\
(9.5-15.5) \\
\end{array}$ \\
\hline $36-47$ & 3,594 & 37,390 & $\begin{array}{c}9.6 \\
(9.3-9.9) \\
\end{array}$ & 3,613 & 39,610 & $\begin{array}{c}9.1 \\
(8.8-9.4) \\
\end{array}$ & $\begin{array}{c}0.5 \\
(0.1-0.9) \\
\end{array}$ & $\begin{array}{c}5.1 \\
(0.8-9.4) \\
\end{array}$ \\
\hline $48-59$ & 1,498 & 37,265 & $\begin{array}{c}4.0 \\
(3.8-4.2) \\
\end{array}$ & 1,423 & 38,967 & $\begin{array}{c}3.6 \\
(3.5-3.8) \\
\end{array}$ & $\begin{array}{c}0.4 \\
(0.1-0.6) \\
\end{array}$ & $\begin{array}{c}9.2 \\
(2.4-15.9) \\
\end{array}$ \\
\hline $0-59$ & 33,807 & 185,369 & $\begin{array}{c}18.2 \\
(18.1-18.4)\end{array}$ & 30,314 & 195,097 & $\begin{array}{c}15.5 \\
(15.4-15.7)\end{array}$ & $\begin{array}{c}2.7 \\
(2.5-2.9)\end{array}$ & $\begin{array}{c}14.8 \\
(13.5-16.1)\end{array}$ \\
\hline
\end{tabular}

$\mathrm{Cl}$ : confidence intervals; IR: incidence rate; RR: relative risk.

TABLE 3

Estimated burden of clinic visits for all-cause and rotavirus acute gastroenteritis, children aged 0-59 months, Maccabi Health Services database, Israel, 2005-13 $(n=293,783)$

\begin{tabular}{|c|c|c|c|c|c|c|}
\hline Year/period & $\begin{array}{c}\text { AGE } \\
\text { visits }\end{array}$ & RV tests & $\begin{array}{l}\% \text { tests performed } \\
\text { among visits }\end{array}$ & $\%$ RV-positive & $\begin{array}{l}\text { Estimated } \\
\text { RV AGE }\end{array}$ & $\begin{array}{c}\text { Estimated incidence rate } \\
\text { per } 100 \\
\text { of RV AGE }(95 \% \mathrm{Cl})\end{array}$ \\
\hline 2005 & 27,728 & 992 & 3.6 & 22.5 & 5,333 & 2.9 \\
\hline 2006 & 37,664 & 1,649 & 4.4 & 25.7 & 8,356 & 4.6 \\
\hline 2007 & 32,271 & 1,757 & 5.4 & $24 \cdot 3$ & 6,603 & 3.6 \\
\hline 2008 & 36,634 & 2,207 & 6.0 & 19.2 & 5,988 & 3.2 \\
\hline 2010 & 40,964 & 2,814 & 6.9 & 19.6 & 7,409 & 3.8 \\
\hline 2011 & 27,922 & 1,858 & 6.7 & 13.9 & 3,857 & 2.0 \\
\hline 2012 & 30,753 & 2,170 & 7.1 & $5 \cdot 3$ & 1,665 & 0.9 \\
\hline 2013 & 32,266 & 2,305 & 7.1 & 7.0 & 2,340 & 1.2 \\
\hline $\begin{array}{l}\text { Annual average in the pre- } \\
\text { vaccination period } 2005^{-10}\end{array}$ & 33,807 & 1,866 & $5 \cdot 5$ & 20.7 & 6,179 & $3.3(3.25-3.4)$ \\
\hline $\begin{array}{l}\text { Absolute reduction in the } \\
\text { annual average between pre- } \\
\text { and post-vaccination period }\end{array}$ & 3,493 & b & $\mathrm{b}$ & 12.0 & 3,557 & $1.98(1.89-2.08)$ \\
\hline $\begin{array}{l}\text { Average annual reduction } \\
\text { between pre- and post- } \\
\text { vaccination period c }\end{array}$ & $10.3 \%$ & b & b & $57 \cdot 9$ & 57.6 & $59.7(59.8-62.6)$ \\
\hline
\end{tabular}

AGE: acute gastroenteritis; $\mathrm{Cl}$ : confidence intervals; RV: rotavirus.

a Estimated by weighted analysis using the inverse of the sampling fraction as the weights, assigned separately in each sex and age group by month and year.

${ }^{b}$ No reduction in rotavirus testing was found after the introduction of universal rotavirus immunisation; on the contrary, there were 245 tests more in 2011-13 than in 2005-10, corresponding to a $13.0 \%$ increase in rotavirus testing.

c Calculated as: [(Annual average in 2005-10 - annual average 2011-13)/ annual average in 2005-10] 100. 
Average annual clinic visits due to rotavirus acute gastroenteritis before (2005-10) and after (2011-13) introduction of universal rotavirus vaccination, by age, Maccabi Health Services, Israel

\begin{tabular}{|c|c|c|c|c|c|c|c|c|}
\hline \multirow[t]{2}{*}{ Age (months) } & \multicolumn{3}{|c|}{$\begin{array}{l}\text { Before universal rotavirus vaccination } \\
\qquad(2005-10)\end{array}$} & \multicolumn{3}{|c|}{$\begin{array}{l}\text { Universal rotavirus vaccination era } \\
\qquad(2011-13)\end{array}$} & \multicolumn{2}{|c|}{ Reduction } \\
\hline & $\begin{array}{l}\text { Estimated } \\
\text { RV AGEa }\end{array}$ & Population & $\begin{array}{l}\text { Estimated } \\
\text { annual IR of RV } \\
\text { AGE per } 100 \\
(95 \% \mathrm{CI})\end{array}$ & $\begin{array}{l}\text { Estimated } \\
\text { RV AGE }\end{array}$ & Population & $\begin{array}{c}\text { Estimated } \\
\text { annual IR of RV } \\
\text { AGE per } 100 \\
(95 \% \mathrm{Cl})\end{array}$ & $\begin{array}{c}\text { Absolute } \\
\text { reduction in IR } \\
\text { per } 100 \\
(95 \% \mathrm{Cl})\end{array}$ & $\begin{array}{c}\text { \% reduction } \\
\text { In IR } \\
(95 \% \mathrm{Cl})\end{array}$ \\
\hline $0-11$ & 1,588 & 35,495 & $\begin{array}{c}4 \cdot 5 \\
(4 \cdot 3-4 \cdot 7) \\
\end{array}$ & 340 & 37,572 & $\begin{array}{c}0.9 \\
(0.8-1.0)\end{array}$ & $\begin{array}{c}3.6 \\
(3.3-3.8) \\
\end{array}$ & $\begin{array}{c}79.8 \\
(74.6-85.0)\end{array}$ \\
\hline $12-23$ & 3,049 & 37,551 & $\begin{array}{c}8.1 \\
(7.8-8.4) \\
\end{array}$ & 1,290 & 39,337 & $\begin{array}{c}3 \cdot 3 \\
(3.1-3 \cdot 5) \\
\end{array}$ & $\begin{array}{c}4.8 \\
(4.5-5.2) \\
\end{array}$ & $\begin{array}{c}59.6 \\
(55.6-63.7) \\
\end{array}$ \\
\hline $24-59$ & 1,542 & 112,324 & $\begin{array}{c}1.37 \\
(1.33-1.44) \\
\end{array}$ & 991 & 118,188 & $\begin{array}{c}0.83 \\
(0.78-0.89) \\
\end{array}$ & $\begin{array}{c}0.53 \\
(0.44-0.62)\end{array}$ & $\begin{array}{c}38.9 \\
(32.7-45.2)\end{array}$ \\
\hline
\end{tabular}

AGE: acute gastroenteritis; $\mathrm{Cl}$ : confidence intervals; IR: incidence rate; RV: rotavirus.

a Estimated by weighted analysis.

to care such as visits to clinics is similar in the four HMOs in Israel; patients do not pay for these services, which are included in the 'national basket health services', irrespective of HMO. Furthermore, the primary care clinics are widespread across the country, the hospitals are public and access to these hospitals is similar among the four HMOs. In addition, access to vaccines included in the NIP is universal and vaccines are given through widespread maternal and child health clinics, mostly operated by the Ministry of Health.

Data on visits to clinics in community settings were retrieved from the MHS database using the International Statistical Classification of Diseases version 9 (ICD-9) codes of acute gastroenteritis and suspected or proven gastroenteritis [25]. We also collected data on the patient's age, sex and date of visit, which was used to define week, month, calendar year and study period with respect to the introduction of RotaTeq into the NIP. Only upon request from the physician, the community microbiological laboratories examine stool specimens obtained from AGE patients for Shigella, Salmonella, Campylobacter, protozoa or viruses including rotavirus. Rotavirus gastroenteritis is not a notifiable disease in Israel, therefore we used the MHS database. We included data on those laboratory tests for rotavirus antigen that were performed within seven days of the clinic visit.

\section{Data analysis}

The annual age-specific incidence rates of AGE (per 100 capita) and their 95\% confidence intervals ( $\mathrm{Cls}$ ) were calculated for each study period. Laboratory results on rotavirus testing were available only for a sample of $6 \%$ all AGE clinic visits, therefore we employed weighted analysis to estimate the number of clinic visits due to rotavirus AGE in children aged o to 59 months in MHS. The weights were defined separately for sex and age group, in each month and year, as the inverse of the sampling fraction in each of these strata; the sampling fraction was considered as the total number of rotavirus tests divided by the total number of AGE clinic visits in the same stratum. The weights were assigned separately for male and female children in each age group (0-11, 12-23 and 24-59 months) in each month and year (January 2005 through May 2014), i.e. in 678 different strata, because rotavirus testing differed by age, sex and period. Using data available for full calendar years (1 January 2005 to 31 December 2013), we calculated the absolute and relative change (in percentages) for the following parameters: number of $A G E$, number of rotavirus-positive tests, proportion of rotavirus-positive tests, and incidence rate (per 100) of rotavirus-related AGE visits.

We analysed seasonality in the period before universal vaccination and in the universal immunisation era by Pocock's harmonic analysis [26], using weekly counts of AGE clinic visits. In this analysis we used data on clinic visits that occurred from 1 January 2005 through 31 December 2013.

\section{Ethical considerations}

The study protocol was approved by the Institution Review Board of Asuta Medical Center. All data were anonymous and no personal identifying information was available for the study investigators. The study was given an exempt from informed consent given its nature of using historical anonymous data.

\section{Results}

During the study period, a total of 302,445 visits to clinics in the community setting due to AGE were documented. Of these, $54.8 \%$ were male and $45.2 \%$ were female children. The mean age of the patients was 21.1 months (standard deviation: 12.7; range 0-59). There were yearly fluctuations in the incidence rate of $A G E$ clinic visits, which were evident in both sexes and in stratification by age (Table 1 ). These fluctuations were more obvious before the introduction of rotavirus vaccines to the NIP than in the period with universal vaccination (2011-13). Overall, the annual incidence of visits 


\section{FIGURE 1}

Number of weekly clinic visits due to acute gastroenteritis, by age group, Maccabi Health Services, Israel, 2005-14 $(\mathrm{n}=302,445)$

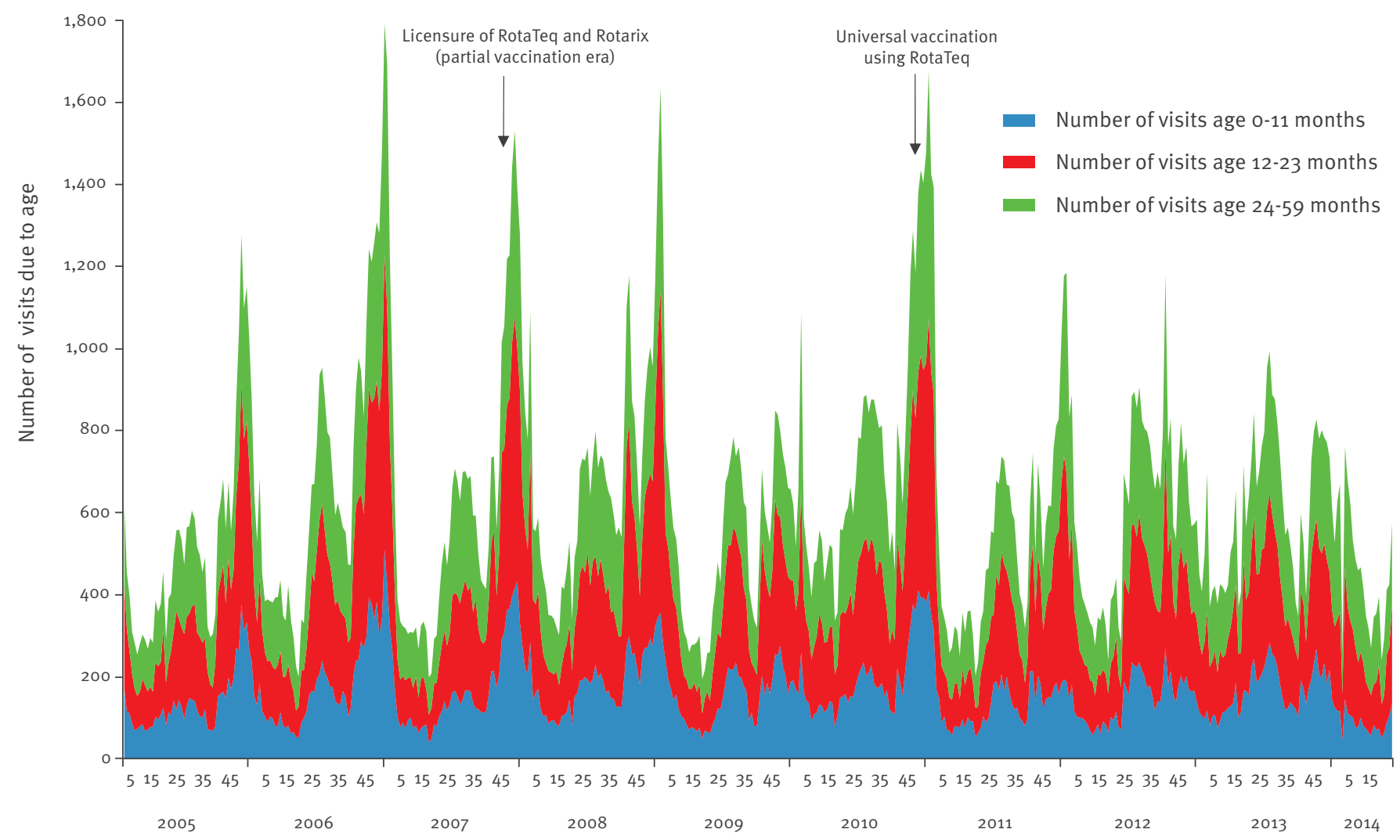

Week/year

AGE: acute gastroenteritis.

to a physician due to AGE followed a downward trend in the period with universal vaccination, ranging from 14.3 to 16.6 (mean: 15.5) per 100 compared with the period from 2005 to 2010 (range: 14.7-21.3; mean 18.2 per 100 (Table 1).

A significant relative reduction of $14.8 \%$ and an absolute risk reduction of 2.7 per 100 in the annual average incidence of AGE clinic visits (Table 2) were found following the introduction of universal rotavirus immunisation. This decline was greater in children aged $0-11$ and 12-23 months than in the older groups (Table 2 ). The magnitude of the change was similar in both sexes (data not shown).

During the period from 1 January 2005 through 16 May $2014,18,133$ tests for the detection of rotavirus antigen were performed within seven days of the AGE clinic visit. Of those, 2,910 (16.0\%) were positive and $15,223(84.0 \%)$ were negative. Rotavirus testing differed by age, sex and period. Infants (o-11 months) and toddlers (12-23 months) were more likely to be tested (odds ratio $(O R)=2.07$ and 1.82 , respectively; $\mathrm{p}$ <0.001) than children aged 24-59 months, testing was performed slightly more often in male than female children $(O R=1.08 ; p<0.001)$, in the post- vs pre-vaccination era $(O R=1.31 ; p<0.001)$, and month-to-month variation was also observed.

In the weighted analysis, using the information on clinic visits attributed to laboratory-confirmed rotavirus AGE from 1 January 2005 till 31 December 2013, we estimated a yearly average of 6,179 rotavirus AGE clinic visits (incidence rate: 3.3 per 100) in the pre-vaccination period compared with 2,621 (incidence rate: 1.34 per 100) in the universal immunisation era, which corresponds to a reduction of $57.6 \%$ in the absolute number and $59.7 \%$ in the incidence rate of rotavirus AGE clinic visits) in MHS (Table 3 ). There was a gradient in the reduction with age: $79.8 \%, 59.6 \%$ and $38.9 \%$ in the age groups $0-11,12-23$ and 24-59 months, respectively (Table 4).

Between 2005 and 2010, there was a clear bimodal seasonality with peaks in spring/summer (warm) and winter (cold) (Figure 1). Except for 2009, the peaks in winter were twice as high as those in summer. This difference between the summer and winter peaks was not apparent in the post-vaccination period. 
Seasonality of weekly clinic visits (aggregated counts) due to acute gastroenteritis in children aged 0-59 months, before and after universal rotavirus vaccination, Maccabi Health Services, Israel, 2005-13 ( $n=293,761)$

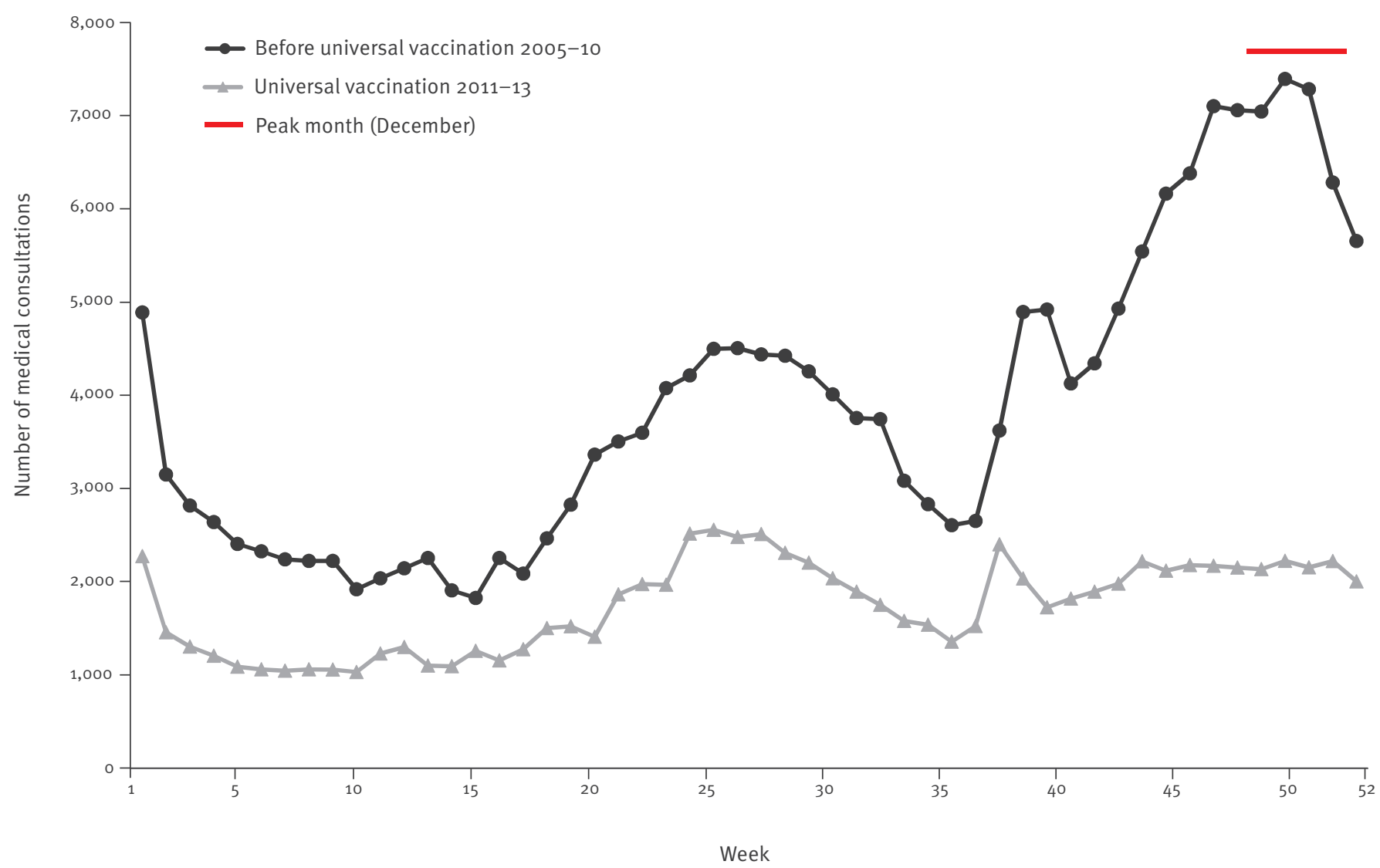

While the timing of the summer peaks were not altered after the introduction of rotavirus immunisation, the winter peaks in the period from 2011 to 2014 occurred earlier (Figure 1). Whereas in the pre-vaccination period, the yearly peak was in December, this changed with the introduction of universal vaccination (Figure 2). In 2011, the winter peak was still observed around December, but this was not evident in 2012 and 2013 (Figures 1 and Figure 3).

Test for seasonality was significant $(p<0.001)$ both in the period before universal immunisation and in the universal vaccination period. Nonetheless, following the introduction of universal rotavirus immunisation, there was a decrease in the average number of weekly AGE clinic visits (from 648 to 583) as well as in the seasonal variation (from $70.9 \%$ to $57.3 \%$ ), while the nonseasonal and random variation increased from $28.4 \%$ to $41.3 \%$, and the standardised ratio of seasonal to random variation consequently decreased from 1.72 to 0.74. Rotavirus positivity followed typical winter seasonality (Figure 3) that coincided with the winter peaks of clinic visits for all-cause AGE (Figure 1, Figure 2 and Figure 3). The percentage of rotavirus-positive samples decreased after the introduction of universal rotavirus vaccination (Figure 3 and Table 3).

\section{Discussion}

In this large population study, we examined the impact of the universal rotavirus immunisation programme using RotaTeq on the burden of mild to moderate AGE and on rotavirus AGE associated with visits to primary care clinics among children younger than five years.

We found that introducing RotaTeq to the NIP in Israel was followed by an impressive reduction (59.7\%) in incidence rate of mild to moderate rotavirus AGE associated with clinic visits (from 3.3 to 1.3 per 100) and a more modest $(14.8 \%)$ decline in clinic visits due to allcause AGE (from 18.2 to 15.5 per 100) in the paediatric population of MHS. The decrease was most notable in children aged o to 23 months, but a reduction was also documented in older children. Rotarix and RotaTeq were licensed in Israel in mid-2007, became available on the market towards the end of 2007 and were administered in the HMO primary care clinics for an approximate fee of ca EUR 88 (ca USD 100) [24]. Parents who purchased rotavirus vaccine received partial reimbursement by their HMO. Therefore, the reduction in rotavirus AGE clinic visits in the older groups could be attributed to partial rotavirus vaccination between 2008 and 2010 , to herd protection conferred by universal rotavirus immunisation of infants or to both. Herd immunity due to rotavirus immunisation was described in 


\section{FIGURE 3}

Rotavirus antigen test results in stools of patients who visited clinics for acute gastroenteritis, by week and year, Maccabi Health Services, Israel, 2005-14 $(n=18,133)$

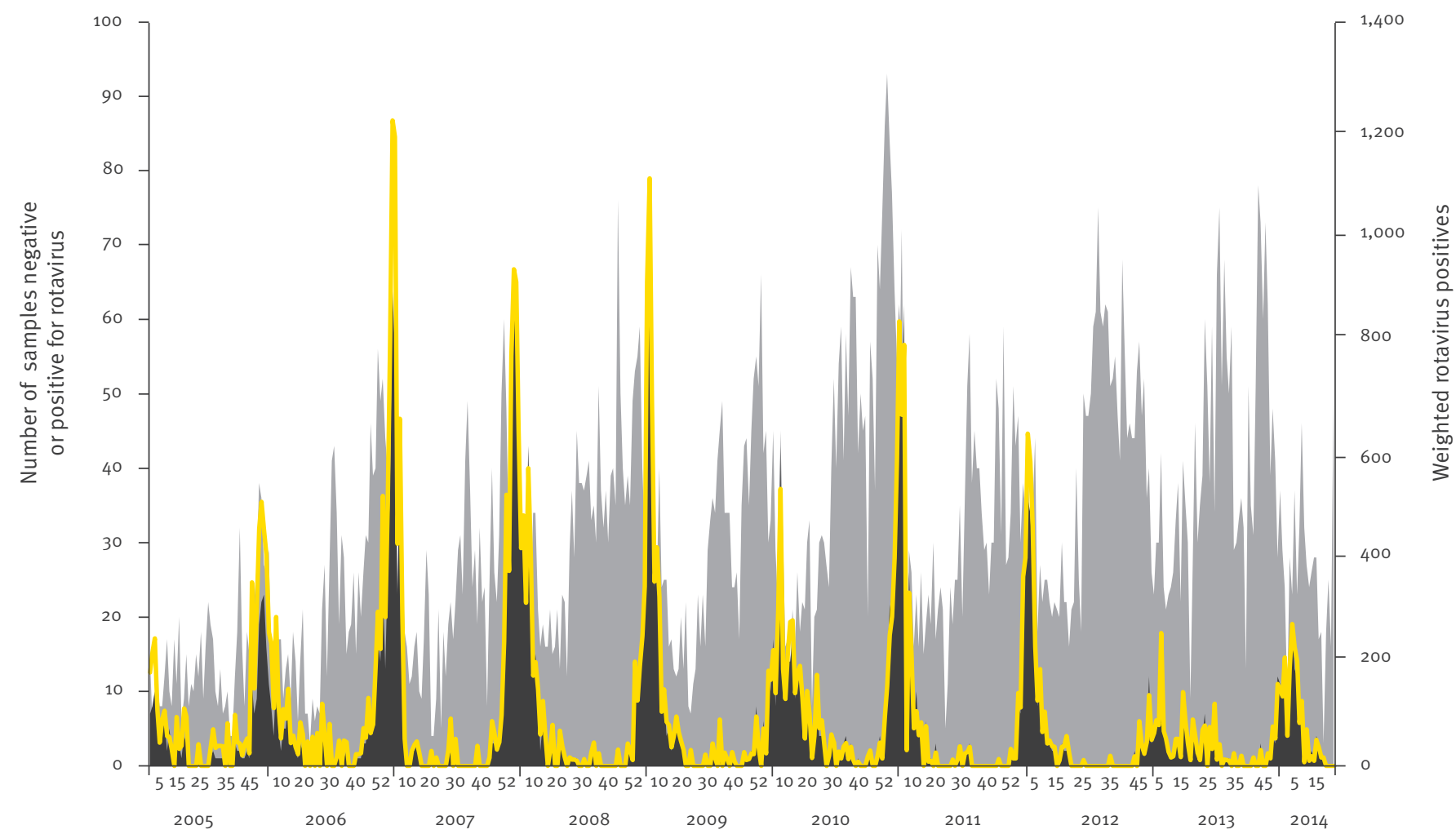

Week/year

Rotavirus-negative

other studies that mostly focused on hospitalisations (reviewed by Patel et al. [12]). The remarkable reduction in clinic visits for rotavirus AGE is probably the result of the high effectiveness of rotavirus vaccines in Israel $[15,24]$, good matching between the predominant circulating rotavirus genotypes and the vaccine strains $[15,23]$, and high vaccination coverage of ca $80 \%$ for three doses. Our findings provide evidence that universal rotavirus immunisation can have a broad impact on reducing the burden of less severe disease in a developed country. So far, the introduction of universal rotavirus immunisation has been slow in middleand high-income countries, including in the European region $[13,14]$. The barriers for implementing universal rotavirus immunisation in many European countries, which have been reviewed recently, include low awareness of the disease burden, perception of unfavourable cost-effectiveness and scepticism regarding the potential benefit of universal vaccination $[13,14]$. Our findings demonstrate a substantial reduction in the rotavirus gastroenteritis burden which was achieved only after the implementation of universal vaccination policy coupled with high immunisation coverage. Such evidence argues against some of the above barriers.
Healthcare utilisation resulting from all-cause diarrhoea was suggested as a possible data source to monitor the impact of rotavirus immunisation on disease burden $[27,28]$. Yet this can be a useful instrument only in settings with good historical medical records. Using healthcare utilisation practices without laboratory information may widely underestimate the true impact of the vaccine. Under these circumstances, the role of existing sentinel laboratory surveillance networks $[29,30]$ becomes crucial.

Before the introduction of universal rotavirus vaccination, there was a clear bimodal seasonality of clinic visits for all-cause AGE, with summer and winter peaks, while clinic visits for rotavirus AGE occurred predominantly in the winter. Following the introduction of RotaTeq to the NIP, the summer peaks remained unaffected while the winter peaks were substantially lower. The change in the winter peaks of AGE clinic visits and rotavirus-positive samples were similar, thus it is plausible to attribute the changes in the all-cause AGE winter peaks to rotavirus immunisation.

Our study may have some limitations. First we considered AGE clinic visits as mild to moderate disease; however, it is possible that a small percentage of 
the patients presented with severe illness and were referred to hospital by their primary care physician. Since disease severity is dynamic, it can be expected that a fraction of the patients who presented with mild disease later developed severe disease and may have been hospitalised. However, it is well documented that only a small fraction (up to $3 \%$ ) of rotavirus gastroenteritides are severe enough to lead to a hospitalisation [3]. Indeed, we have estimated the incidence of rotavirus gastroenteritis hospitalisations in children under the age of five years in the pre-vaccination period in Israel at ca5.7 per 1,000. Applying this figure to the MHS paediatric population shows that up to $3.1 \%$ of clinic visits could have been severe cases which may have resulted in hospitalisations. These data support the assumption that the majority of cases included in our study had mild to moderate gastroenteritis. Moreover, the majority of children who visited a clinic for AGE were not tested for rotavirus. However, knowing the total number of AGE clinic visits and retrieving data on rotavirus antigen testing, which was performed in ca $7 \%$ of all clinic visits, allowed us to conduct a weighted analysis to estimate the impact of rotavirus immunisation on rotavirus AGE clinic visits. It is worth mentioning that we characterised the factors that were significantly associated with rotavirus testing (age, sex, month and year of clinic visit) and that all were taken into account in the weighting procedure. Lastly, the period before universal rotavirus immunisation included three years (2008-10) of partial immunisation in which the uptake of the vaccine was fair (ca $50 \%$ in MHS) [24]. Therefore the current study is likely to underestimate vaccine impact.

The strengths of our study include the use of a computerised HMO database which includes a large representative sample of ca $25 \%$ of the Israeli population. Information in this database is gathered as part of routine clinical care of MHS members. We included data that were gathered over a nine-year period on over 0.3 million recorded clinic visits. This enabled us to detect the true impact of the universal rotavirus immunisation programme on the primary care setting. All laboratory tests in MHS are performed in one central laboratory (Mega Laboratory) and to our knowledge, the rotavirus testing method did not change during the time covered in this study.

\section{Conclusion}

Universal rotavirus vaccination in Israel was followed by a considerable decline in rotavirus AGE clinic visits and, to a lesser extent, in all-cause AGE clinic visits in children younger than five years. The decrease was greatest in children under the age of two years but was also evident in older children, possibly resulting from herd protection and/or partial rotavirus immunisation of the older cohorts during the pre-universal rotavirus immunisation era. Using data on healthcare utilisation may widely underestimate the true impact of the vaccine and may not be valuable in the long term, especially given the altered rotavirus seasonality after inclusion of universal rotavirus immunisation. Therefore, continued laboratory-based surveillance for rotavirus is warranted. These results have implications for global public health and policy making.

\section{Conflict of interest}

None declared.

\section{Authors' contributions}

K.M, D.C, G.C and V.S planned the study. G.C and V.S, collected the data. K.M, D.C, S.G, T.Z and E.A analysed the data. K.M, D.C and G.C wrote the first draft of the manuscript. All authors contributed the interpretation of the results and finalising the manuscript. K.M and G.C have equal contribution as first authors.

\section{References}

1. ParasharUD, HummelmanEG, BreseeJS, MillerMA, GlassRI. Global illness and deaths caused by rotavirus disease in children.Emerg Infect Dis. 2003;9(5):565-72. DOI: 10.3201/ eido905.020562 PMID: 12737740

2. MusherDM, MusherBL. Contagious acute gastrointestinal infections.N Engl J Med. 2004;351(23):2417-27. DOI: 10.1056/ NEJMra041837 PMID: 15575058

3. GlassRI, ParasharUD, BreseeJS, TurciosR, FischerTK, WiddowsonMA, et al. Rotavirus vaccines: current prospects and future challenges. Lancet. 2006;368(9532):323-32. DOI: 10.1016/S0140-6736(06)68815-6 PMID: 16860702

4. TateJE, BurtonAH, Boschi-PintoC, SteeleAD, DuqueJ, ParasharUD, et al. . 2008 estimate of worldwide rotavirusassociated mortality in children younger than 5 years before the introduction of universal rotavirus vaccination programmes: a systematic review and meta-analysis. Lancet Infect Dis. 2012;12(2):136-41. DOI: 10.1016/S14733099(11)70253-5 PMID: 22030330

5. MelliezH, BoellePY, BaronS, MoutonY, YazdanpanahY. [Morbidity and cost of rotavirus infections in France]. Med Mal Infect. 2005;35(10):492-9. French. DOI: 10.1016/j. medmal.2005.08.007 PMID: 16316731

6. Ogilviel, KhouryH, GoetghebeurMM, El KhouryAC, GiaquintoC. Burden of community-acquired and nosocomial rotavirus gastroenteritis in the pediatric population of Western Europe: a scoping review.BMC Infect Dis. 2012;12(1):62. DOI: 10.1186/1471-2334-12-62 PMID: 22429601

7. LeeBP, AzimiPH, StaatMA, LouieL, ParadaE, BerkeT, et al. Nonmedical costs associated with rotavirus disease requiring hospitalization. Pediatr Infect Dis J. 2005;24(11):984-8. DOI: 10.1097/01.inf.0000183754.29707.cd PMID: 16282934

8. Ruiz-PalaciosGM, Pérez-Schaell, VelázquezFR, AbateH, BreuerT, ClemensSC, et al. Safety and efficacy of an attenuated vaccine against severe rotavirus gastroenteritis. N Engl J Med. 2006;354(1):11-22. DOI: 10.1056/NEJMoa052434 PMID: 16394298

9. VesikariT, MatsonDO, DennehyP, Van DammeP, SantoshamM, RodriguezZ, et al. . Safety and efficacy of a pentavalent human-bovine $\left(\mathrm{WC}_{3}\right)$ reassortant rotavirus vaccine. $\mathrm{N}$ Engl J Med. 2006;354(1):23-33. DOI: 10.1056/NEJMoa052664 PMID: 16394299

10. PatelMM, ParasharUD. Assessing the effectiveness and public health impact of rotavirus vaccines after introduction in immunization programs.J Infect Dis. 2009;200(s1) Suppl 1;S291-9. DOI: 10.1086/605059 PMID: 19817612

11. LopmanBA, PayneDC, TateJE, PatelMM, CorteseMM, ParasharUD. Post-licensure experience with rotavirus vaccination in high and middle income countries; 2006 to 2011.Curr Opin Virol. 2012;2(4):434-42. DOI: $10.1016 / j$. coviro.2012.05.002 PMID: 22749491

12. PatelMM, GlassR, DesaiR, TateJE, ParasharUD. Fulfilling the promise of rotavirus vaccines: how far have we come since licensure?Lancet Infect Dis. 2012;12(7):561-70. DOI: 10.1016/ S1473-3099(12)70029-4 PMID: 22742639

13. ParezN, GiaquintoC, Du RoureC, Martinon-TorresF, SpoulouV, Van DammeP, et al. Rotavirus vaccination in Europe: drivers and barriers. Lancet Infect Dis. 2014;14(5):416-25. DOI: 10.1016/S1473-3099(14)70035-0 PMID: 24758998 
14. HuppertzH, BorteM, SchusterV, GiaquintoC, VesikariT. Report of the Third European Expert Meeting on Rotavirus Vaccination: Progress in rotavirus universal mass vaccination in Europe.Vaccine. 2014;32(34):4243-8. DOI: 10.1016/j. vaccine.2014.05.029 PMID: 24852720

15. MuhsenK, ShulmanL, KasemE, RubinsteinU, Shachter], KremerA, et al. Effectiveness of rotavirus vaccines for prevention of rotavirus gastroenteritis-associated hospitalizations in Israel: a case-control study. Hum Vaccin. 2010;6(6):450-4. DOI: 10.4161/hv.6.6.11759 PMID: 20448471

16. Paulke-KorinekM, Rendi-WagnerP, KundiM, KronikR, KollaritschH. Universal mass vaccination against rotavirus gastroenteritis: impact on hospitalization rates in austrian children.Pediatr Infect Dis J. 2010;29(4):319-23.PMID: 19935446

17. LeinoT, OllgrenJ, SaloH, TiihonenP, KilpiT. First year experience of rotavirus immunisation programme in Finland.Vaccine. 2012;31(1):176-82. DOI: 10.1016/j.vaccine.2012.10.068 PMID: 23122991

18. BraeckmanT, Van HerckK, MeyerN, PirçonJY, SorianoGabarróM, HeylenE, et al. . Effectiveness of rotavirus vaccination in prevention of hospital admissions for rotavirus gastroenteritis among young children in Belgium: case-control study.BM). 2012;345(augo8 1):e4752. DOI: 10.1136/bmj.e4752 PMID: 22875947

19. RichardsonV, Hernandez-PichardoJ, Quintanar-SolaresM, Esparza-AguilarM, JohnsonB, Gomez-AltamiranoCM, et al. Effect of rotavirus vaccination on death from childhood diarrhea in Mexico. N Engl J Med. 2010;362(4):299-305. DOI: 10.1056/NEJMoa0905211 PMID: 20107215

20. do CarmoGM, YenC, Cortes), SiqueiraAA, de OliveiraWK, Cortez-EscalanteJJ, et al. Decline in diarrhea mortality and admissions after routine childhood rotavirus immunization in Brazil: a time-series analysis. PLoS Med. 2011;8(4):e1001024. DOI: 10.1371/journal.pmed.1001024 PMID: 21526228

21. NolanSM, PrasadP, FiksAG, ZaoutisT, TenhaveTR, CoffinSE. Effect of rotavirus vaccine on reducing acute gastroenteritis in a large outpatient pediatric network. Arch Pediatr Adolesc Med. 2012;166(3):232-9. DOI: 10.1001/archpediatrics.2011.628 PMID: 22393181

22. LeshemE, MoritzRE, CurnsAT, ZhouF, TateJE, LopmanBA, et al. Rotavirus vaccines and health care utilization for diarrhea in the United States (2007-2011). Pediatrics. 2014;134(1):15-23. DOI: $10.1542 /$ peds.2013-3849 PMID: 24913793

23. MuhsenK, ShulmanL, RubinsteinU, KasemE, KremerA, GorenS, et al. Incidence, characteristics, and economic burden of rotavirus gastroenteritis associated with hospitalization of israeli children <5 years of age, 2007-2008. J Infect Dis. 2009;200(s1) Suppl 1;S254-63. DOI: 10.1086/605425 PMID: 19817606

24. MuhsenK, ChodickG, GorenS, ShalevV, CohenD. The uptake of rotavirus vaccine and its effectiveness in preventing acute gastroenteritis in the community.Vaccine. 2010;29(1):91-4. DOI: 10.1016/j.vaccine.2010.10.010 PMID: 20969927

25. World Health Organization (WHO). International statistical classification of diseases and related health problems. Ninth revision. Geneva: WHO; 1995.

26. PocockSJ. Harmonic-Analysis Applied to SeasonalVariations in Sickness Absence.J R Stat Soc Ser C Appl Stat. 1974;23(2):103-20.

27. AmadorJJ, VasquezJ, OrozcoM, PedreiraC, MalespinO, De OliveiraLH, et al. Rotavirus disease burden, Nicaragua 20012005: defining the potential impact of a rotavirus vaccination program. Int J Infect Dis. 2010;14(7):e592-5. DOI: 10.1016/j. ijid.2009.08.014 PMID: 20022778

28. NgaboF, GateraM, KaremaC, DonnenP, LepageP, ParasharUD, et al. Can routinely collected national data on childhood morbidity and mortality from diarrhea be used to monitor health impact of rotavirus vaccination in Africa? Examination of pre-vaccine baseline data from Rwanda. Pediatr Infect Dis J. 2014;33(Suppl 1):S89-93. DOI: 10.1097/ INF.0000000000000054 PMID: 24343621

29. KotloffKL, NataroJP, BlackwelderWC, NasrinD, FaragTH, PanchalingamS, et al. Burden and aetiology of diarrhoeal disease in infants and young children in developing countries (the Global Enteric Multicenter Study, GEMS): a prospective, case-control study. Lancet. 2013;382(9888):209-22. DOI: 10.1016/S0140-6736(13)60844-2 PMID: 23680352

30. Centers for Disease Control and Prevention (CDC),. Rotavirus surveillance--worldwide, 2001-2008.MMWR Morb Mortal Wkly Rep. 2008;57(46):1255-7.PMID: 19023263 